Research Article

\title{
Alpha Ideals and the Space of Prime Alpha Ideals in Universal Algebras
}

\author{
Gezahagne Mulat Addis \\ Department of Mathematics, University of Gondar, Gondar, Ethiopia \\ Correspondence should be addressed to Gezahagne Mulat Addis; buttu412@yahoo.com
}

Received 23 July 2020; Revised 19 May 2021; Accepted 21 May 2021; Published 9 June 2021

Academic Editor: Kit C. Chan

Copyright ( $\odot 2021$ Gezahagne Mulat Addis. This is an open access article distributed under the Creative Commons Attribution License, which permits unrestricted use, distribution, and reproduction in any medium, provided the original work is properly cited.

The purpose of this paper is to study $\alpha$-ideals in a more general context, in universal algebras having a constant 0 . Several characterizations are obtained for an ideal $I$ of an algebra $A$ to be an $\alpha$-ideal. It is shown that the class of all $\alpha$-ideals of an algebra $A$ forms an algebraic lattice. Prime $\alpha$-ideals and several related properties are investigated. Some properties of the spectral space of prime $\alpha$-ideals equipped with the hull-kernel topology are derived.

\section{Introduction}

$\alpha$-ideals were first studied by Cornish [1] for a class of distributive lattices as a generalization of annihilator ideals. Later on, the notion of $\alpha$-ideals was extended to the class of 0 -distributive lattices by Pawar and Khopade [2], to the class of almost distributive lattices by Rao and Rao [3], to the class of $C$-algebras by Rao [4], and more generally to arbitrary posets by Mokbel [5]. Jayaram [6] studied prime $\alpha$-ideals in 0 -distributive lattices and he topologized the class of all prime $\alpha$-ideals in 0-distributive lattices. Bigard [7] has also studied $\alpha$-ideals in the context of lattice-ordered groups. Instead of annulets, he used the dual lattice of carriers.

Cornish, in his paper [1], studied $\alpha$-ideals in a distributive lattice $L$, by using the lattice $A_{0}(L)$ of all annulets of the form $(x)^{*}$ (where $(x)^{*}=\{a \in L: a \wedge x=0\}$ ) which is a sublattice of the Boolean algebra of all annihilator ideals in $L$. Cornish has defined two operators $\alpha$ and $\overleftarrow{\alpha}$ as follows: for an ideal $J$ in $L$,

$$
\alpha(J)=\left\{(x)^{*}: x \in J\right\},
$$

is a filter in $A_{0}(L)$ and, conversely, for a filter $F$ of $A_{0}(L)$,

$$
\overleftarrow{\alpha}(F)=\left\{x \in L:(x)^{*} \in F\right\},
$$

is an ideal in $L$. It is observed that the map $J \longmapsto \overleftarrow{\alpha} \alpha(J)$ forms a closure operator on the class of all ideals of $L$ and $\alpha$-ideals in $L$ are defined to be those ideals which are closed with respect to this closure operator, i.e., $J$ is an $\alpha$-ideal of $L$ if

$$
J=\overleftarrow{\alpha} \alpha(J)=\left\{x \in L:(x)^{*}=(y)^{*} \text { for some } y \in J\right\} .
$$

In fact, this property can be equivalently expressed in the following way: $J$ is an $\alpha$-ideal of $L$ if and only if $(x)^{* *} \subseteq J$ for all $x \in J$. Cornish has observed that, by using the structure of $A_{0}(L)$, results can be transferred to give information on the ideal structure of $L$. The most interesting result of this type is that $L$ is a generalized Stone lattice if and only if each prime ideal contains a unique prime $\alpha$-ideal. Moreover, necessary and sufficient conditions are also given for a distributive lattice $L$ with 0 to be disjunctive using $\alpha$-ideals.

In [8], Chajda and Halas have introduced the notion of annihilators in general universal algebras having a constant 0 . In [9], we propose a new approach to study annihilators in universal algebras by the use of commutator terms. In the present paper, we continue our study and define $\alpha$-ideals as a generalization of those annihilator ideals in universal algebras. We have also studied the basic topological properties of the space of $\alpha$-prime ideals in universal algebras. This is a reasonable abstraction of the theory of $\alpha$-ideals of distributive lattices to general universal algebras with abstract finitary operations. The results of this paper are important to extend the properties of distributive lattices to other classes 
of algebras such as groups, rings, $\mathrm{BCK} / \mathrm{BCI}$ algebras, $\mathrm{MV}$ algebras, and Hilbert algebras.

\section{Preliminaries}

This section contains some definitions and results which will be used in this paper. For the standard concepts in universal algebras, we refer to [10-12]. Throughout this paper, $A \in \mathscr{K}$, where $\mathscr{K}$ is a class of algebras of a fixed type $\mathfrak{F}$ and assume that there is an equationally definable constant in all algebras of $\mathscr{K}$ denoted by 0 . For a positive integer $n$, we write $\vec{a}$ to denote the $n$-tuple $\left\langle a_{1}, a_{2}, \ldots, a_{n}\right\rangle \in A^{n}$. The $n$-ary terms of type $\mathfrak{F}$ are formal expressions obtained in finitely many steps by the following process:

(1) The variables $x_{1}, \ldots, x_{n}$ are $n$-ary terms of type $\mathfrak{F}$

(2) If $m \in Z_{0}, t_{1}, \ldots, t_{m}$ are $n$-ary terms of type $\mathfrak{F}$ and $f \in \mathfrak{F}_{m}$, then $f\left(t_{1}, \ldots, t_{m}\right)$ is also a term of type $\mathfrak{F}$

Definition 1 (see [13]). An $(n+m)$-ary term $p(\vec{x}, \vec{y})$ is said to be an ideal term in $\vec{y}$ if

$$
p\left(a_{1}, \ldots, a_{n}, 0,0, \ldots, 0\right)=0,
$$

for all $a_{1}, \ldots, a_{n} \in A$. A nonempty subset $I$ of $A$ will be called an ideal of $A$ if $p(\vec{a}, \vec{b}) \in I$ for all $\vec{a} \in A^{n}, \vec{b} \in I^{m}$ and any ideal term $p(\vec{x}, \vec{y})$ in $\vec{y}$.

We denote the class of all ideals of $A$ by $i(A)$. It is easy to check that the intersection of any family of ideals of $A$ is an ideal. So, for a subset $S \subseteq A$, there always exists a smallest ideal of $A$ containing $S$ which we call the ideal of $A$ generated by $S$ and it is denoted by $\langle S\rangle$. Note that $x \in\langle S\rangle$ if and only if $x=p\left(a_{1}, \ldots, a_{n}, b_{1}, \ldots, b_{m}\right)$ for some $a_{1}, \ldots, a_{n} \in A$, and $b_{1}, \ldots, b_{m} \in S$ where $p(\vec{x}, \vec{y})$ is an $(n+m)$-ary ideal term in $\vec{y}$. If $S=\{a\}$, then we write $\langle a\rangle$ instead of $\langle S\rangle$. In this case, $x \in\langle a\rangle$ if and only if $x=p\left(a_{1}, \ldots, a_{n}, a\right)$ for some $a_{1}, \ldots, a_{n} \in A$, where $p(\vec{x}, \vec{y})$ is an $(n+1)$-ary ideal term in $\vec{y}$.

Definition 2 (see [13]). A class $\mathscr{K}$ of algebras is called ideal determined if every ideal $I$ is the zero-congruence class of a unique congruence relation denoted by $I^{\delta}$. In this case, the map $I \longmapsto I^{\delta}$ defines an isomorphism between the lattice of ideals and the congruence lattice of $A$.

Definition 3 (see $[13,14])$. A term $t(\vec{x}, \vec{y}, \vec{z})$ is said to be a commutator term in $\vec{y}, \vec{z}$ if it is an ideal term in $\vec{y}$ and an ideal term in $\vec{z}$.

Definition 4 (see [13]). In an ideal determined variety, the commutator $[I, J]$ of ideals $I$ and $J$ is the zero-congruence class of the commutator congruence $\left[I^{\delta}, J^{\delta}\right]$.

Throughout this paper, $\mathscr{K}$ is assumed to be an idealdetermined variety and each $A \in \mathscr{K}$ has a $c$-unit.

Theorem 1 (see $[13,14])$. Let $I$ and $J$ be ideals of $A \in \mathscr{K}$. Then

$$
\begin{aligned}
{[I, J]=} & \left\{t(\vec{a}, \vec{i}, \vec{j}): \vec{a} \in A^{n}, \vec{i} \in I^{m} \text { and } \vec{j} \in J^{k}\right. \\
& \text { where } t(\vec{x}, \vec{y}, \vec{z}) \text { is a commutator term in } \vec{y}, \vec{z}\} .
\end{aligned}
$$

For subsets $H$ and $G$ of $A,[H, G]$ denotes the product $[\langle H\rangle,\langle G\rangle]$. In particular, for $a, b \in A,[\langle a\rangle,\langle b\rangle]$ is denoted by $[a, b]$. The following lemma gives some properties of the commutator of ideals. Further details on ideals of universal algebras can be found in [15-17].

Lemma 1 (see [14]). For each $I, J, K \in i(A)$, we have the following:

(1) $[I, J]=[J, I]$

(2) $I \subseteq K$ implies $[I, J] \subseteq[K, J]$

(3) The commutator of ideals is distributive over arbitrary joins of ideals

Definition 5 (see [14]). A nonzero element $u$ in $A$ is said to be a formal unit if $A=\langle u\rangle$, i.e., $A$ is generated by $u$ as an ideal.

A cyclic group, a ring with unity, a bounded lattice, and an almost distributive lattice with maximal elements are examples of an algebra having a formal unit [9].

Definition 6. A nonzero element $u \in A$ is a called a commutator unit (or $c$-unit for short) in $A$ if $[a, u]=\langle a\rangle$ for all $a \in A[9]$.

Example 1

(1) In a ring $R$ with unity, the elements 1 and -1 are $c$-unit

(2) In a bounded distributive lattice $L$, the largest element 1 is a $c$-unit

(3) In a bounded Hilbert algebra $A$, the least element 0 is a $c$-unit

(4) Every maximal element in an almost distributive lattice is a $c$-unit

\section{Annihilators}

This section contains some important results on annihilators and annihilator ideals of universal algebras taken from.

Definition 7 (see [9]). Let $I \in i(A)$. For any subset $S$ of $A$, we define

$$
(S: I)=\{a \in A:[a, S] \subseteq I\} .
$$

We call $(S: I)$ the relative annihilator of $S$ with respect to $I$. For a subset $S$ of $A$, the annihilator of $S$ denoted by $S^{*}$ is defined to be

$$
S^{*}=(S:(0)) \text {. }
$$


If $S=\{a\}$, then we denote $S^{*}$ by $(a)^{*}$, i.e.,

$(a)^{*}=\{b \in A:[a, b]=\langle 0\rangle\}$.

It is observed that $S^{*}$ is an ideal of $A$ and $S^{*}=\langle S\rangle^{*}$ for all $S \subseteq A$. Also, it can be verified that $(0)^{*}=A$ and $A^{*}=(0)$.

Lemma 2. For any subsets $S$ and $T$ of $A$,

(1) $S^{*}=\cap_{a \in S}(a) *$

(2) $S \subseteq S^{* *}$

(3) $S^{* * *}=S^{*}$

(4) $S \subseteq T \Longrightarrow T^{*} \subseteq S^{*}$

(5) $(S \cup T)^{*}=S^{*} \cap T^{*}$

An element $a$ in a ring $R$ is said to be idempotent if $a^{2}=a$. By imitating this property to the general case of universal algebras, we define the following.

Definition 8. An element $a \in A$ is called idempotent if

$$
[a, a]=\langle a\rangle .
$$

If all elements of $A$ are idempotent, then we call $A$ a commutator idempotent algebra (or a $c$-idempotent algebra for short). In other words, the commutator of ideals in $A$ is idempotent.

Boolean rings and more generally lattices with least element 0 are the most natural examples of $c$-idempotent algebras. One can also verify that regular rings (a ring $R$ is regular if for each $x \in R$ there exists $a \in R$ such that $x a x=x$ ) are examples of $c$-idempotent algebras. One of the most important properties of $c$-idempotent algebras is that the commutators of ideals coincide with their intersection. Further, the separation axiom holds in $c$-idempotent algebras.

Definition 9. An ideal $I$ of $A$ is called an annihilator ideal if $I=S^{*}$ for some nonempty subset $S$ of $A$.

It is immediate from the definition that an ideal $I$ of $A$ is an annihilator ideal if and only if $I^{* *}=I$. We denote the class of all annihilator ideals of $A$ by $i^{*}(A)$. If $A$ is a $c$-idempotent algebra, then it is proved that $i^{*}(A)$ forms a Boolean algebra.

Definition 10. An ideal $I$ of $A$ is called a dense ideal if $I^{*}=(0)$; otherwise, $I$ is called a nondense ideal.

\section{4. $\alpha$ - Ideals}

In this section, we study $\alpha$-ideals in universal algebras in a more general context.

Notation 1. We write $F \subset \subset A$ to say that $F$ is a finite subset of $A$.

Definition 11. An ideal $I$ of $A$ is called an $\alpha$-ideal if

$$
F^{* *} \subseteq I \text { for each } F \subset \subset I \text {. }
$$

We denote by $i_{\alpha}(A)$ the class of all $\alpha$-ideals of $A$. $\alpha(A)$.

It is easy to check that $\langle 0\rangle$ and $A$ belong to the class

Remark 1. If $A$ is a distributive lattice, then this definition coincides to the definition proposed by Cornish [1].

Lemma 3. Every annihilator ideal of $A$ is an $\alpha$-ideal.

Remark 2. The converse of Lemma 3 does not hold in general. For instance, in an infinite algebra $A$ with 0 , every proper dense $\alpha$-ideal is not an annihilator ideal.

Lemma 4. In a finite algebra $A$, every $\alpha$-ideal is an annihilator ideal.

Theorem 2. An ideal I of $A$ is an $\alpha$-ideal if and only if

$$
I=\bigcup_{F \subset \subset I} F^{* *} \text {. }
$$

Proof. Suppose that $I$ is an $\alpha$-ideal. Then $F^{* *} \subseteq I$ for all finite subsets $F$ of $I$, which gives

$$
\bigcup_{F \subset \subset I} F^{* *} \subseteq I \text {. }
$$

Also, if $a \in I$, then $F=\{a\}$ is a finite subset of $I$ such that $a \in F^{* *}$. So, the other inclusion holds. The converse part is straightforward.

Definition 12. For each $I \in i(A)$, let us define a set $\alpha(I)$ as follows:

$$
\alpha(I)=\left\{x \in A: F^{*} \subseteq(x)^{*} \text { for some } F \subset \subset I\right\} .
$$

In the following lemma, we give another description for the set $\alpha(I)$ which will be useful to prove Theorem 2 .

Lemma 5. For each $I \in i(A)$, we have

$$
\alpha(I)=\cup\left\{H \in i(A): F^{*} \subseteq H^{*} \text { for some } F \subset \subset I\right\} .
$$

Proof. Let us define two sets $K$ and $G$ as follows:

$$
\begin{aligned}
& K=\left\{x \in A: F^{*} \subseteq(x)^{*} \text { for some } F \subset \subset I\right\}, \\
& G=\cup\left\{H \in i(A): F^{*} \subseteq H^{*} \text { for some } F \subset \subset I\right\} .
\end{aligned}
$$

We show that $K=G$. Since $(a)^{*}=\langle a\rangle^{*}$ for all $a \in A$, it holds immediately that $K \subseteq G$. To prove the other inclusion, let $a \in G$. Then there is $H \in i(A)$ and $F \subset \subset I$ such that $a \in H$ and $F^{*} \subseteq H^{*}$, which gives $H^{*} \subseteq(a)^{*}$. So, $a \in K$ and therefore the equality holds.

Lemma 6. For each $I, J \in i(A)$, we have the following:
(1) $I \subseteq \alpha(I)$
(2) $I \subseteq J \Longrightarrow \alpha(I) \subseteq \alpha(J)$
(3) $\alpha(\alpha(I))=\alpha(I)$

Theorem 3. For each $I \in i(A), \alpha(I)$ is an ideal of $A$. 
Proof. Let $\vec{b}=\left\langle b_{1}, b_{2}, \ldots, b_{m}\right\rangle$ be an $m$-tuple of elements from $\alpha(I)$. Then, for each $b_{i}$, there exist $H_{1}, \ldots, H_{m} \in i(A)$ and finite subsets $F_{1}, \ldots, F_{m}$ of $I$ such that $b_{i} \in H_{i}$ and $F_{i}^{*} \subseteq H_{i}^{*}$. Put $F=\cup_{i=1}^{m} F_{i}$ and $H=\vee_{i=1}^{m} H_{i}$. Then $F$ is a finite subset of $I$ and $H \in i(A)$ such that $F^{*}=\cap_{i=1}^{m} F_{i}^{*}$ and $H^{*}=$ $\cap_{i=1}^{m} H_{i}^{*}$ and $b_{1}, b_{2}, \ldots, b_{m} \in H$. Since $F_{i} \subseteq F, F^{*} \subseteq F_{i}^{*} \subseteq H_{i}^{*}$ for all $i$. So, $F^{*} \subseteq \cap_{i=1}^{m} H_{i}^{*}=H^{*}$. That is, $H$ is an ideal of $A$ such that $F^{*} \subseteq H^{*}$. Hence, $H \subseteq \alpha(I)$. Now if $\vec{a} \in A^{n}$ and $p(\vec{x}, \vec{y})$ is an ideal term in $\vec{y}$, then $p(\vec{a}, \vec{b}) \in H \subseteq \alpha(I)$. Therefore, $\alpha(I)$ is an ideal of $I$.

Theorem 4. For each $I \in i(A), \alpha(I)$ is the smallest $\alpha$-ideal of A containing $I$.

Proof. We first show that $\alpha(I)$ is an $\alpha$-ideal. Let us put

$$
G=\cup\left\{H \in i(A): F^{*} \subseteq H^{*} \text { for some } F \subset \subset I\right\} .
$$

Let $F \subset \subset G$. We show that $F^{* *} \subseteq G$. If $F=\varnothing$, then $F^{* *}=(0) \subseteq G$. Suppose that $F \neq \varnothing$, and let $F=\left\{a_{1}, \ldots, a_{n}\right\}$. Then, for each $a_{i}$, there exist $H_{1}, \ldots, H_{n} \in i(A)$ and finite subsets $F_{1}, \ldots, F_{n}$ of $I$ such that $a_{i} \in H_{i}$ and $F_{i}^{*} \subseteq H_{i}^{*}$ for all $i$. Put $E=\cup_{i=1}^{n} F_{i}$ and $H=\vee_{i=1}^{n} H_{i}$. Then $E$ is a finite subset of $I$, and $H \in i(A)$ such that $a_{1}, \ldots, a_{n} \in H$ and $E^{*}=\cap_{i=1}^{n} F_{i}^{*}$ and $H^{*}=\cap_{i=1}^{n} H_{i}^{*}$. So, $E^{*} \subseteq F_{i}^{*} \subseteq H_{i}^{*}$ for all $i$, which gives $E^{*} \subseteq H^{*}$. Since $F \subseteq H$, we have $F^{* *} \subseteq H^{* *}$. It remains to show that $H^{* *} \subseteq G$. If we put $K=H^{* *}$, then $K$ is an ideal of $A$ such that $K^{*}=H^{*}$. Since $E$ is a finite subset of $I$ with $E^{*} \subseteq K^{*}$, we get $K \subseteq G$. Therefore $G=\alpha(I)$ is an $\alpha$-ideal. It is also observed in Lemma 6 that $I \subseteq \alpha(I)$. Now let $J$ be any other $\alpha$-ideal of $A$ such that $I \subseteq J$ and let $a \in \alpha(I)$. Then there exists $H \in i(A)$ and $F \subset \subset I$ such that $a \in H$ and $F^{*} \subseteq H^{*}$. So, $H^{* *} \subseteq F^{* *}$. Also, since $F \subseteq I \subseteq J$ and $J$ is an $\alpha$-ideal, we get $F^{* *} \subseteq J$. Now consider the following:

$$
a \in H \subseteq H^{* *} \subseteq F^{* *} \subseteq J .
$$

Hence, $\alpha(I) \subseteq J$ and therefore $\alpha(I)$ is the smallest $\alpha$-ideal containing $I$.

Theorem 5. An ideal I of $A$ is an $\alpha$-ideal if and only if $I=\alpha(I)$.

Proof. Suppose that $I$ is an $\alpha$-ideal. Since the other inclusion is trivial, we proceed to show that $\alpha(I) \subseteq I$. Let $a \in \alpha(I)$. Then there exists $H \in i(A)$ and $F \subset \subset I$ such that $a \in H$ and $F^{*} \subseteq H^{*}$. We have the following:

$$
a \in H \subseteq H^{* *} \subseteq F^{* *} \subseteq I .
$$

Therefore, $\alpha(I) \subseteq I$ and hence the equality holds. The converse part is straightforward.

From Lemma 6, it can be deduced that the map $\alpha: i(A) \longrightarrow i(A): I \longmapsto \alpha(I)$ is a closure operator on $i(A)$ and $\alpha$-ideals of $A$ are precisely the closed elements of $i(A)$ with respect to this closure operator.

Theorem 6. An ideal I of $A$ is an $\alpha$-ideal if and only if for each finite subset $F$ of $A$ and any subset $E$ of $A, F^{*}=E^{*}$ and $F \subseteq I$ together imply $E \subseteq I$.
Proof. Suppose that $I$ is an $\alpha$-ideal and let $F \subset \subset A$ and $E \subseteq A$ such that $F^{*}=E^{*}$. Then $F^{* *}=E^{* *}$. If $F \subseteq I$, then $F^{* *} \subseteq I$ and hence $E^{* *} \subseteq I$. So $E \subseteq I$. Conversely, suppose that the above condition is satisfied. Let $F \subset \subset I$. We show that $F^{* *} \subseteq I$. Put $E=F^{* *}$. Then $E \subseteq A$ such that $F^{*}=E^{*}$. As $F \subset \subset I$, we get $E \subseteq I$. Hence, the result holds.

Theorem 7. Let $A$ be a c-idempotent algebra and $P$ be a prime ideal of $A$. If $P$ is nondense, then it is an $\alpha$-ideal.

Proof. Suppose that $P$ is nondense. Then there exists a nonzero $a \in P^{*}$ which implies that $P=(a)^{*}$. Thus $P$ is an annihilator ideal and hence it follows from Lemma 3 that $P$ is an $\alpha$-ideal.

Theorem 8. The collection $\left\langle i_{\alpha}(A), \subseteq\right\rangle$ forms a complete lattice.

Proof. It is enough to show that $i_{\alpha}(A)$ is closed under arbitrary intersection. Let $\left\{J_{\lambda}\right\}_{\lambda \in \Delta}$ be an indexed family of $\alpha$-ideals in $A$. Put $J=\cap_{\lambda \in \Delta} J_{\lambda}$. If $\Delta$ is empty, then $J=A$ and hence it is an $\alpha$-ideal. Assume that $\Delta$ is nonempty. Then $J$ is an ideal of $A$. Moreover, if $F$ is a finite subset of $J$, then it can be easily observed that $F^{* *} \subseteq J$ and therefore it is an $\alpha$-ideal.

Note also that, for any $I, J \in i_{\alpha}(A)$, their supremum is given by

$$
I \sqcup J=\alpha(I \vee J)
$$

For a nonempty set $X$, remember from [12] that a family $\mathfrak{E}$ of subsets of $X$ will be called a closure system in $\mathscr{P}(X)$ if it forms a complete lattice together with the usual inclusion order. Furthermore, a closure system $\mathfrak{c}$ is an algebraic lattice if and only if every chain in $\mathfrak{E}$ has supremum in $\mathfrak{E}$.

Theorem 9. The collection $\left\langle i_{\alpha}(A), \subseteq\right\rangle$ is an algebraic lattice.

Proof. It is enough to show that every chain in $i_{\alpha}(A)$ has an upper bound in $i_{\alpha}(A)$. Let $\left\{J_{\lambda}\right\}_{\lambda \in \Delta}$ be a chain in $i_{\alpha}(A)$. Put $J=\cup_{\lambda \in \Delta} J_{\lambda}$. We first show that $J$ is an ideal. Let $p(\vec{x}, \vec{y})$ be an $(n+m)$-ary ideal term in $\vec{y}, a_{1}, \ldots, a_{n} \in A$, and $b_{1}, \ldots, b_{m} \in J$. Then, there exist $\lambda_{1}, \ldots, \lambda_{m} \in \Delta$ such that each $b_{i} \in J_{\lambda_{i}}$. Since the family $\left\{J_{\lambda}\right\}_{\lambda \in \Delta}$ is a chain, we can find $\lambda \in \Delta$ with $b_{1}, \ldots, b_{m} \in J_{\lambda}$. With $J_{\lambda}$ being an ideal of $A$, it holds that

$$
p\left(a_{1}, \ldots, a_{n}, b_{1}, \ldots, b_{m}\right) \in J_{\lambda} \subseteq J .
$$

Therefore, $J$ is an ideal of $A$. It remains to show that $J$ is an $\alpha$-ideal. Let $F$ be a finite subset of $F$. If $F=\varnothing$, then $F^{* *}=(0) \subseteq J$. Assume that $F$ is nonempty and let $F=\left\{a_{1}\right.$, $\left.\ldots, a_{n}\right\}$. Then, there exist $\lambda_{1}, \ldots, \lambda_{n} \in \Delta$ such that each $a_{i} \in J_{\lambda_{i}}$. Since the family $\left\{J_{\lambda}\right\}_{\lambda \in \Delta}$ is a chain, we can find $\lambda \in \Delta$ with $a_{1}, \ldots, a_{n} \in J_{\lambda}$, i.e., $F \subset \subset J_{\lambda}$. With $J_{\lambda}$ being an $\alpha$-ideal of $A$, it follows that $F^{* *} \subseteq J_{\lambda} \subseteq J$. Therefore, $J$ is an $\alpha$-ideal. This completes the proof.

It can be deduced from the above theorem that the closure operator $J \longmapsto \alpha(J)$ is in fact an algebraic closure operator. Moreover, the compact elements in $i_{\alpha}(A)$ are those finitely generated $\alpha$-ideals of $A$. 
Definition 13 (see [14]). A subset $M$ of $A$ is called an $m$-system if, for all $a, b \in M,[a, b] \cap M \neq \varnothing$.

In the following lemma, we give another description for $m$-systems for our purpose.

Lemma 7. A nonempty subset $M$ of $A$ is an $m$-system if and only if, for any nonempty subsets $S_{1}$ and $S_{2}$ of $M$,

$$
M \cap\left[S_{1}, S_{2}\right] \neq \varnothing \text {. }
$$

Proof. Suppose that $M$ is an $m$-system. Let $S_{1}, S_{2}$ be nonempty subsets of $M$. Choose $a \in S_{1}$ and $b \in S_{2}$. Then $[a, b] \subseteq\left[S_{1}, S_{2}\right]$. Since $a, b \in M, M \cap[a, b] \neq \varnothing$, which gives $M \cap\left[S_{1}, S_{2}\right] \neq \varnothing$. The converse part is straightforward.

Definition 14 . For an $m$-system $M$ of $A$, let us define a set $0(M)$ as follows:

$$
0(M)=\{x \in A:[x, S]=(0) \text { for some } S \subseteq M\} .
$$

Lemma 8. For each $m$-system $M$ of $A$,

$$
0(M)=\cup\{H \in i(A):[H, S]=(0) \text { for some } S \subseteq M\} .
$$

Theorem 10. For each $m$-system $M$ of $A$, the set $0(M)$ is an ideal of $A$.

Proof. Let $\vec{b}=\left\langle b_{1}, b_{2}, \ldots, b_{k}\right\rangle$ be a $k$-tuple of elements from $0(M)$. Then for each $b_{i}$ there exist $H_{1}, \ldots, H_{k} \in i(A)$ and nonempty subsets $S_{1}, \ldots, S_{k}$ of $M$ such that $b_{i} \in H_{i}$ and $\left[H_{i}, S_{i}\right]=(0)$ for all $i$. If we put $H=\vee_{i=1}^{k} H_{i}$, then $H \in i(A)$ such that $b_{1}, b_{2}, \ldots, b_{k} \in H$. On the other hand, let us define a sequence $M_{1}, M_{2}, \ldots M_{k}$ as follows:

$$
\begin{aligned}
M_{1} & =S_{1} \text { and } \text { for } 1 \leq j<k, \\
M_{j+1} & =\left[M_{j}, S_{j+1}\right] \cap M .
\end{aligned}
$$

Since $M$ is an $m$-system, each $M_{j}$ is a nonempty subset of $M$ such that $M_{j} \subseteq S_{j}$. Moreover,

$$
M_{k} \subseteq M_{k-1} \subseteq \cdots \subseteq M_{2} \subseteq M_{1} .
$$

Now consider the following:

$$
\left[H, M_{k}\right]=\left[\mathrm{V}_{i=1}^{k} H_{i}, M_{k}\right]=\vee_{i=1}^{k}\left[H_{i}, M_{k}\right] \subseteq \vee_{i=1}^{k}\left[H_{i}, S_{i}\right]=(0) \text {. }
$$

Therefore, $H \subseteq 0(M)$. Now, if $\vec{a} \in A^{n}$ and $p(\vec{x}, \vec{y})$ is an ideal term in $\vec{y}$, we get $p(\vec{a}, \vec{b}) \in H$. So, $p(\vec{a}, \vec{b}) \in 0(M)$. Therefore $0(M)$ is an ideal.

Theorem 11. For each $m$-system $M$ of $A$, the set $0(M)$ is an $\alpha$-ideal of $A$.

Proof. Let $F=\left\{a_{1}, a_{2}, \ldots, a_{n}\right\}$ be a finite subset of $0(M)$. Then there exist $H_{1}, \ldots, H_{n} \in i(A)$ and nonempty subsets $S_{1}, \ldots, S_{n}$ of $M$ such that $a_{i} \in H_{i}$ and $\left[H_{i}, S_{i}\right]=(0)$ for all $i$. If we let $H=\vee_{i=1}^{m} H_{i}$, then $H \in i(A)$ such that $a_{1}, a_{2}, \ldots, a_{m} \in H$. On the other hand, let us define a sequence $M_{1}, M_{2}, \ldots, M_{n}$ as follows:

$$
\begin{aligned}
M_{1} & =S_{1} \text { and for } 1 \leq k<n, \\
M_{k+1} & =\left[M_{k}, S_{k+1}\right] \cap M .
\end{aligned}
$$

Since $m$ - is an $M$-system, each $M_{k}$ is a nonempty subset of $M$ such that $M_{k} \subseteq S_{k}$. Moreover,

$$
M_{n} \subseteq M_{n-1} \subseteq \cdots \subseteq M_{2} \subseteq M_{1} .
$$

Now consider the following:

$\left[H, M_{n}\right]=\left[V_{i=1}^{n} H_{i}, M_{n}\right]=V_{i=1}^{n}\left[H_{i}, M_{n}\right] \subseteq V_{i=1}^{n}\left[H_{i}, S_{i}\right]=(0)$.

That is, $\left[H, M_{n}\right]=(0)$, where $M_{n}$ is a nonempty subset of $M$. So, $H \subseteq M_{n}^{*}$, which gives $H^{* *} \subseteq M_{n}^{* * *}=M_{n}^{*}$. Thus, $\left[H^{* *}, M_{n}\right]=(0)$ and hence $H^{* *} \subseteq 0(M)$. Since $F \subseteq H$, we have $F^{* *} \subseteq H^{* *}$. So, $F^{* *} \subseteq 0(M)$. Therefore, $0(M)$ is an $\alpha$-ideal.

Definition 15. For each prime ideal $P$ of $A$, let us define $0^{\prime}(P)$ as follows:

$0^{\prime}(P)=\{a \in A:[a, S]=(0)$ for some $S \subseteq A$ with $S \cap P=\varnothing\}$.

If we let $M=A-P$, then $M$ is an $m$-system of $A$ such that $0^{\prime}(P)=0(M)$. So, it follows from Theorems 10 and 11 that $0^{\prime}(P)$ is an $\alpha$-ideal of $A$. Moreover, if $P$ is minimal prime ideal, then $0^{\prime}(P)$ is an $\alpha$-ideal containing $P$.

Theorem 12. Let $A$ be a c-idempotent algebra. Then the following are equivalent:

(1) Every ideal I of $A$ is an $\alpha$-ideal

(2) Every prime ideal $P$ of $A$ is an $\alpha$-ideal

Proof. $\quad(1) \Longrightarrow(2)$ is obvious. We proceed to show $(2) \Longrightarrow(1)$. Assume (2). Let $I$ be an ideal of $A$. It is enough if we show that $\alpha(I) \subseteq I$. Suppose not. Then there exists $x \in \alpha(I)$ but $x \notin I$. Since $A$ is $c$-idempotent, we can apply Zorn's lemma to obtain a prime ideal $P$ of $A$ such that $I \subseteq P$ and $x \notin P$. So $\alpha(I) \subseteq \alpha(P)=P$. This is a contradiction. Thus, $\alpha(I) \subseteq I$ and hence $I$ is an $\alpha$-ideal.

Remark 3. If the algebra we are working on is a distributive lattice, then one (and hence all) of the conditions of Theorem 12 is necessary and sufficient condition for $A$ to be disjunctive.

\section{The Space of Prime $\alpha$-Ideals}

Definition 16. By a prime $\alpha$-ideal of $A$, we mean an $\alpha$-ideal $P$ of $A$ satisfying the condition

$$
[I, J] \subseteq P \Longrightarrow I \subseteq P \text { or } J \subseteq P,
$$

for all $I, J \in i(A)$. 
Theorem 13. Let $I$ be an $\alpha$-ideal of $A$ and $M$ be an $m$-system of $A$ such that $I \cap M=\varnothing$. Then there exists a prime $\alpha$-ideal $P$ of $A$ such that $I \subseteq P$ and $P \cap M=\varnothing$.

Proof. Let us put

$$
\mathfrak{Q}=\left\{J \in i_{\alpha}(A): I \subseteq J \text { and } J \cap M=\varnothing\right\} .
$$

Clearly $\mathfrak{Q}$ is a nonempty family of $\alpha$-ideals of $A$ satisfying the hypothesis of Zorn's lemma. So $\mathfrak{L}$ has a maximal element, say $P$. Our aim is to show that $P$ is prime. Suppose not. Then there exist $a, b \in A-P$ such that $[a, b] \subseteq P$. If we put $J=P \sqcup \alpha(\langle a\rangle)$ and $K=P \sqcup \alpha(\langle b\rangle)$, then $J$ and $K$ are $\alpha$-ideals of $A$ properly containing $P$. By the maximality of $P$ in $\mathfrak{Q}$, both $J$ and $K$ do not belong to $\mathfrak{Q}$. So $J \cap M \neq \varnothing$ and $K \cap M \neq \varnothing$. Choose $x, y \in M$ such that $x \in J$ and $y \in K$. Consider the following:

$$
\begin{aligned}
{[x, y] \subseteq[J, K]=} & {[P \sqcup \alpha(\langle a\rangle), P \sqcup \alpha(\langle b\rangle)] } \\
= & {[P, P] \sqcup[P, \alpha(\langle a\rangle)] \sqcup[P, \alpha(\langle b\rangle)] \sqcup[\alpha(\langle a\rangle),} \\
& \cdot \alpha(\langle b\rangle)] \subseteq P .
\end{aligned}
$$

Since $P \cap M=\varnothing$, it follows that $[x, y]=\varnothing$. This is a contradiction. Therefore, $P$ is prime.

Corollary 1. Let I be an $\alpha$-ideal of $A$ and $a \in A$ such that $a \notin$ I. If $A$ is c-idempotent, then there exists a prime $\alpha$-ideal $P$ of $A$ such that $I \subseteq P$ and $a \notin P$.

Proof. Since $A$ is $c$-idempotent, every singleton $\{a\}$ is an $m$-system. So, the proof follows from Theorem 13 .

Theorem 14. Let $A$ be a c-idempotent algebra. Then, for any $\alpha$-ideal I of $A$, we have

$$
I=\cap\{P: P \text { is a prime } \alpha-\text { ideal of } A \text { and } I \subseteq P\} .
$$

Proof. Let us put

$$
H=\cap\{P: P \text { is a prime } \alpha-\text { ideal of } A \text { and } I \subseteq P\} .
$$

Clearly, $I \subseteq H$. To prove the other inclusion, let $x \notin I$. Then, by Corollary 1, there exists a prime $\alpha$-ideal $P$ of $A$ such that $I \subseteq P$ and $x \notin P$, i.e., $x \notin H$. Thus, $H \subseteq I$ and hence the equality holds.

Theorem 15. Let $A$ be a c-idempotent algebra. Then, for any $\alpha$-ideal I of $A$, we have

$$
I^{*}=\cap\{P: P \text { is a prime } \alpha-\text { ideal of } A \text { and } I \nsubseteq P\} .
$$

Proof. Let us put

$$
G=\cap\{P: P \text { is a prime } \alpha \text {-ideal of } A \text { and } I \nsubseteq P\} .
$$

Let $x \in A$ and $P$ be a prime $\alpha$-ideal of $A$ with $I \nsubseteq P$. If $x \in I^{*}$, then $[x, I]=(0) \subseteq P$. Since $P$ is prime and $I \nsubseteq P$ it follows that $x \in P$, i.e., $x \in G$, and hence $I^{*} \subseteq G$. To prove the other inclusion, let $x \in A$ and $P$ be any prime $\alpha$-ideal of $A$ with $I \nsubseteq P$. If $x \notin I^{*}$, then $[x, I] \neq(0)$. Choose a nonzero $y \in[x, I]$. By Corollary 1 , there exists a prime $\alpha$-ideal $P$ of $A$ such that $y \notin P$, which gives $[x, I] \nsubseteq P$. Then $x \notin P$ and hence $x \notin G$. Therefore, $G \subseteq I^{*}$ and the equality holds.

In the rest of our work, $A$ is assumed to be $c$-idempotent. Let us now give the following notations:

$$
\mathscr{P}_{\alpha}(A)=\{P: P \text { is a prime } \alpha-\text { ideal of } A\} .
$$

For each subset $S$ of $A$, let

$$
\begin{aligned}
D_{\alpha}(S) & =\left\{P \in \mathscr{P}_{\alpha}(A): S \nsubseteq P\right\}, \\
V_{\alpha}(S) & =\left\{P \in \mathscr{P}_{\alpha}(A): S \subseteq P\right\} .
\end{aligned}
$$

For each $a \in A$, we write $D_{\alpha}(a)$ instead of $D_{\alpha}(\{a\})$. For any subset $S$ of $A$, one can verify that

$$
D_{\alpha}(\alpha(\langle S\rangle))=D_{\alpha}(\langle S\rangle)=D_{\alpha}(S) .
$$

Lemma 9. For any $\alpha$-ideal I of $A$, we have

$$
D_{\alpha}(I)=\bigcup_{x \in I} D_{\alpha}(x)=\bigcup_{x \in I} D_{\alpha}\left((x)^{* *}\right) .
$$

Proof. The equality $D_{\alpha}(I)=\cup_{x \in I} D_{\alpha}(x)$ holds trivially. For the other equality, it is enough to show that $D_{\alpha}(x)=D_{\alpha}\left((x)^{* *}\right)$ for all $x \in A$. For any prime $\alpha$-ideal $P$ of $A$, one can verify that $x \in P$ if and only if $(x)^{* *} \subseteq P$.

Lemma 10. The following conditions hold for all $I, J \in i(A)$ :
(1) $I \subseteq J \Longrightarrow D_{\alpha}(I) \subseteq D_{\alpha}(J)$
(2) $D_{\alpha}(I \cap J)=D_{\alpha}(I) \cap D_{\alpha}(J)=D_{\alpha}([I, J])$
(3) $D_{\alpha}(I \vee J)=D_{\alpha}(I) \cup D_{\alpha}(J)$
(4) $D_{\alpha}\left(\vee_{\lambda \in \Delta} I_{\lambda}\right)=\cup_{\lambda \in \Delta} D_{\alpha}\left(I_{\lambda}\right)$

Lemma 11. The following conditions are equivalent for any ideals $I$ and $J$ of $A$ :
(1) $D_{\alpha}(I)=D_{\alpha}(J)$
(2) $V_{\alpha}(I)=V_{\alpha}(J)$
(3) $\alpha(I)=\alpha(J)$

Theorem 16. The family $\mathfrak{I}_{\alpha}=\left\{D_{\alpha}(I)\right.$ : I is an $\alpha$-ideal of $\left.A\right\}$ forms a topology on $\mathscr{P}_{\alpha}(A)$.

Proof. Clearly, both $\varnothing$ and $\mathscr{P}_{\alpha}(A)$ belong to the family $\mathfrak{I}_{\alpha}$. Moreover, it follows from (2) and (4) of Lemma 10 that $\mathfrak{I}_{\alpha}$ is closed under finite intersection and arbitrary unions and thus it is a topology on $\mathscr{P}_{\alpha}$.

Definition 17. We call the topology $\mathfrak{I}_{\alpha}$ the $\alpha$-prime spectrum of $A$ and sometimes it may be denoted as $\operatorname{Spec}_{\alpha}(A)$.

Lemma 12. The subfamily $\mathscr{B}_{\alpha}=\left\{D_{\alpha}(x): x \in A\right\}$ constitutes a base for the topology $\mathfrak{I}_{\alpha}$. 
Proof. Let $I$ be $n$-ideal of $A$ and $P \in D_{\alpha}(I)$. Then there exists $x \in I$ such that $x \notin P$, i.e., $P \in D_{\alpha}(x) \subseteq D_{\alpha}(I)$. Thus $\mathscr{B}_{\alpha}$ is a base for $\mathfrak{I}_{\alpha}$.

Theorem 17. Each $D_{\alpha}(x)$ is a compact open subset of $\mathscr{P}_{\alpha}(A)$.

Proof. Let $\left\{D_{\alpha}\left(x_{\lambda}\right)\right\}_{\lambda \in \Delta}$ be a basic open cover for $D_{\alpha}(x)$, i.e.,

$$
D_{\alpha}(x) \subseteq \bigcup_{\lambda \in \Delta} D_{\alpha}\left(x_{\lambda}\right) \text {. }
$$

Let $I$ be an ideal of $A$ generated by $\left\{x_{\lambda}: \lambda \in \Delta\right\}$. Then $D_{\alpha}(x) \subseteq D_{\alpha}(I)=D_{\alpha}(\alpha(I))$. Our aim is to show that $x \in \alpha(I)$. Suppose on the contrary that $x \notin \alpha(I)$. Then there exists a prime $\alpha$-ideal $P$ of $A$ such that $\alpha(I) \subseteq P$ and $x \notin P$, i.e., $P \in D_{\alpha}(x)$ but $P \notin D_{\alpha}(\alpha(I))$, which is a contradiction. Thus, $x \notin \alpha(I)$. There exists a finite subset $F$ of $I$ such that $F^{*} \subseteq(x)^{*}$. Since $I$ is generated by $\left\{x_{\lambda}: \lambda \in \Delta\right\}$, there exist $k \in N$ and $\lambda_{1}, \ldots, \lambda_{k} \in \Delta$ such that $F \subseteq\left\langle x_{\lambda_{1}}, \ldots, x_{\lambda_{k}}\right\rangle$. If we put $E=\left\{x_{\lambda_{1}}, \ldots, x_{\lambda_{k}}\right\}$, then $E$ is a finite subset of $I$ such that $E^{*} \subseteq F^{*} \subseteq(x)^{*}$. Now we show that

$$
D_{\alpha}(x) \subseteq \bigcup_{i=1}^{k} D_{\alpha}\left(x_{\lambda_{i}}\right)
$$

For any prime $\alpha$-ideal $P$ of $A$, if $x \notin P$, then $(x)^{* *} \nsubseteq P$ and hence $E^{* *} \nsubseteq P$, i.e.,

$$
D_{\alpha}(x) \subseteq D_{\alpha}(\langle E\rangle)=\bigcup_{i=1}^{k} D_{\alpha}\left(x_{\lambda_{i}}\right) .
$$

This completes the proof.

Corollary 2. If $A$ has a dense element, then $\mathscr{P}_{\alpha}(A)$ is compact.

Proof. If $A$ has a dense element say $d$, then $D_{\alpha}(d)=\mathscr{P}_{\alpha}(A)$. It follows from Theorem 17 that $\mathscr{P}_{\alpha}(A)$ is compact.

Theorem 18. If every finitely generated ideal in $A$ is a principal ideal, then $\mathscr{B}_{\alpha}$ is the set of all compact open subsets of $\mathscr{P}_{\alpha}(A)$.

Proof. Suppose that every finitely generated ideal in $A$ is a principal ideal. It is proved in Theorem 17 that every element in $\mathscr{B}_{\alpha}$ is compact and open. Let $H$ be a compact open subset of $\mathscr{P}_{\alpha}(A)$. Then there is some $\alpha$-ideal $I$ of $A$ such that

$$
H=D_{\alpha}(I)=\cup_{x \in I} D_{\alpha}(x) .
$$

Since $H$ is compact, there exist $x_{1}, \ldots, x_{m} \in I$ such that

$$
H=\bigcup_{i=1}^{m} D_{\alpha}\left(x_{i}\right)=D_{\alpha}\left(\left\langle\left\{x_{i}: 1 \leq i \leq m\right\}\right\rangle\right)
$$

It follows from our assumption that there is some $y \in A$ such that $H=D_{\alpha}(y)$. Hence proved.

Corollary 3. If every finitely generated ideal in $A$ is a principal ideal and $\mathscr{P}_{\alpha}(A)$ is compact, then $A$ has dense elements.
Theorem 19. There is a lattice isomorphism between the lattice of all $\alpha$-ideals of $A$ and the lattice of all open subsets of $\mathscr{P}_{\alpha}(A)$.

Proof. One can easily verify that the map $I \longmapsto D_{\alpha}(I)$ is an isomorphism between the lattice of all $\alpha$-ideals of $A$ and the lattice of open subsets of $\mathscr{P}_{\alpha}(A)$.

We denote the closure, interior, and exterior of a subfamily $\mathscr{F}$ of $\mathscr{P}_{\alpha}(A)$ by $\operatorname{cl}(\mathscr{F})$, int $(\mathscr{F})$, and $\operatorname{ext}(\mathscr{F})$, respectively. The following theorem shows that the topology $\mathfrak{T}_{\alpha}$ on $\mathscr{P}_{\alpha}(A)$ is the hull-kernel topology.

Theorem 20. For any subfamily $\mathscr{F}$ of $\mathscr{P}_{\alpha}(A)$, $c l(\mathscr{F})=V_{\alpha}\left(\cap_{H \in \mathscr{F}} H\right)$.

Proof. Suppose that $P \in \operatorname{cl}(\mathscr{F})$. Then every neighborhood of $P$ meets $\mathscr{F}$. If we put $J=\cap_{H \in \mathscr{F}} H$, then $J$ is an $\alpha$-ideal of $A$ such that $J \subseteq H$ for all $H \in \mathscr{F}$. Our claim is to show that $J \subseteq P$. Suppose not. Then $P \in D_{\alpha}(J)$ and hence there is some $H \in \mathscr{F}$ such that $J \nsubseteq H$, which is a contradiction. Thus, $P \in V_{\alpha}(J)$. Conversely, suppose that $P \in V_{\alpha}(J)$ and let $I$ be any $\alpha$-ideal of $A$ with $I \nsubseteq P$. We show that $D_{\alpha}(I) \cap \mathscr{F} \neq \varnothing$. If not, then $I \subseteq H$ for all $H \in \mathscr{F}$ and hence $I \subseteq P$, which is a contradiction. Therefore, $P \in \operatorname{cl}(\mathscr{F})$ and the equality holds.

Theorem 21. For any $\alpha$-ideal I of $A$,

$$
\operatorname{ext}\left(\operatorname{cl}\left(D_{\alpha}(I)\right)\right)=D_{\alpha}\left(I^{*}\right) \text {. }
$$

Proof. By Theorem 20, we have

$$
\begin{aligned}
\operatorname{cl}\left(D_{\alpha}(I)\right) & =V_{\alpha}\left(\cap_{P \in D_{\alpha}(A)} P\right) \\
& =V_{\alpha}\left(\cap_{I \nsubseteq P} P\right) \\
& =\left\{Q \in \mathscr{P}_{\alpha}(A): \cap_{I \nsubseteq P} P \subseteq Q\right\} \\
& \left.=\left\{Q \in \mathscr{P}_{\alpha}(A): I^{*} \subseteq Q\right\} \text { (by Theorem } 22\right) V_{\alpha}\left(I^{*}\right) .
\end{aligned}
$$

So $\mathscr{P}_{\alpha}(A)-\operatorname{cl}\left(D_{\alpha}(I)\right)=D_{\alpha}\left(I^{*}\right)$. Now consider the following:

$$
\begin{aligned}
\operatorname{ext}\left(\operatorname{cl}\left(D_{\alpha}(I)\right)\right) & =\operatorname{int}\left(\mathscr{P}_{\alpha}(A)-\operatorname{cl}\left(D_{\alpha}(I)\right)\right) \\
& =\operatorname{int}\left(D_{\alpha}\left(I^{*}\right)\right) \\
& =D_{\alpha}\left(I^{*}\right) .
\end{aligned}
$$

An open subset of a topological space $X$ is called an open domain if it is identical with the interior of its closure and it is called semiregular if it is a union of open domains.

Theorem 22. For an $\alpha$-ideal I of $A, D_{\alpha}(I)$ is an open domain if and only if $I=I^{* *}$.

Proof. It follows from the above theorem that $\operatorname{cl}\left(D_{\alpha}(I)\right)=V_{\alpha}\left(I^{*}\right)$. Now consider the following: 


$$
\begin{aligned}
\operatorname{int}\left(\operatorname{cl}\left(D_{\alpha}(I)\right)\right) & =\operatorname{int}\left(V_{\alpha}\left(I^{*}\right)\right) \\
& =\operatorname{int}\left(\mathscr{P}_{\alpha}(A)-D_{\alpha}\left(I^{*}\right)\right) \\
& =\mathscr{P}_{\alpha}(A)-\operatorname{cl}\left(D_{\alpha}\left(I^{*}\right)\right) \mathscr{P}_{\alpha}(A)-V_{\alpha}\left(I^{* *}\right) \\
& =D_{\alpha}\left(I^{* *}\right) .
\end{aligned}
$$

If $I=I^{* *}$, then $\operatorname{int}\left(\operatorname{cl}\left(D_{\alpha}(I)\right)\right)=D_{\alpha}\left(I^{* *}\right)=D_{\alpha}(I)$ and hence $D_{\alpha}(I)$ is an open domain. Conversely, if $\operatorname{int}\left(\operatorname{cl}\left(D_{\alpha}(I)\right)\right)=D_{\alpha}(I)$, then $D_{\alpha}\left(I^{* *}\right)=D_{\alpha}(I)$ and it follows from Lemma 13 that $I=I^{* *}$.

Corollary 4. For any $\alpha$-ideal I of $A, D_{\alpha}(I)$ is semiregular. In particular, if $A$ has a dense element, then $\mathscr{P}_{\alpha}(A)$ is semiregular.

Theorem 23. If every finitely generated ideal in $A$ is principal, then the following conditions are equivalent:

(1) For each $x \in A$, there exists $y \in A$ such that $(x)^{* *}=(y)^{*}$

(2) Exterior of every compact open subset of $\mathscr{P}_{\alpha}(A)$ is compact

Proof. Suppose that (1) holds. Let $Y$ be a compact open subset of $\mathscr{P}_{\alpha}(A)$. By Theorem 18, $Y=D_{\alpha}(x)$ for some $x \in A$. Now $\operatorname{ext}(Y)=\operatorname{ext}\left(D_{\alpha}(x)\right)=D_{\alpha}\left((x)^{*}\right)$. By (1), there exists $y \in A$ such that $(x)^{*}=(y)^{* *}$. Thus, $\operatorname{ext}(Y)=D_{\alpha}\left((x)^{*}\right)=D_{\alpha}\left((y)^{* *}\right)=D_{\alpha}((y))$. Again, by Theorem 18, ext $(Y)$ is compact. Conversely assume (2). Let $x \in A$. Then $D_{\alpha}(x)$ and its exterior are compact, i.e., $D_{\alpha}\left((x)^{*}\right)$ is compact and open. By Theorem 18, there exists $y \in A$ such that $D_{\alpha}\left((x)^{*}\right)=D_{\alpha}(y)=D_{\alpha}\left((y)^{* *}\right)$. It follows from Lemma 11 that $(x)^{*}=(y)^{* *}$ and this completes the proof.

If $A$ has formal unit, then it was proved in [14] that every proper ideal $I$ of $A$ is contained in a maximal ideal $M$ of $A$. This can be proved by applying Zorn's lemma.

Theorem 24. If $A$ has a formal unit, then $\mathscr{P}_{\alpha}(A)$ is a $T_{1}$-space if and only if every $\alpha$-prime ideal of $A$ is maximal.

Proof. Suppose that $\mathscr{P}_{\alpha}(A)$ is $T_{1}$. Let $P$ be an $\alpha$-prime ideal of $A$, which is not maximal. Since $A$ has a formal unit, there exists a maximal ideal $M$ of $A$ such that $P \subsetneq M$. As $\mathscr{P}_{\alpha}(A)$ is a $T_{1}$-space, there exist basic open sets $D_{\alpha}(a)$ and $D_{\alpha}(b)$ such that $P \in D_{\alpha}(a), M \in D_{\alpha}(b), P \notin D_{\alpha}(b)$, and $M \notin D_{\alpha}(a)$, i.e., $a \in M-P$ and $b \in P-M=\varnothing$. This is a contradiction. Thus, $P$ is maximal. Conversely, suppose that every prime $\alpha$-ideal of $A$ is maximal. Let $P$ and $Q$ be two distinct elements of $\mathscr{P}_{\alpha}(A)$. Then, by assumption, $P \nsubseteq Q$ and $Q \nsubseteq P$. This implies that there exist $a, b \in A$ such that $a \in P-Q$ and $b \in Q-P$. Then we have $P \in D_{\alpha}(b)-D_{\alpha}(a)$ and $Q \in D_{\alpha}(a)-D_{\alpha}(b)$. Thus, $\mathscr{P}_{\alpha}(A)$ is a $T_{1}$-space.

Theorem 25. If $\mathscr{P}_{\alpha}(A)$ is a Hausdorff space containing more than one element, then there exist $a, b \in A$ such that

$$
\mathscr{P}_{\alpha}(A)=D_{\alpha}(a) \cup D_{\alpha}(b) \cup V_{\alpha}(\langle\{a, b\}\rangle) .
$$

Proof. Suppose that $\mathscr{P}_{\alpha}(A)$ is Hausdorff. Let $P$ and $Q$ be distinct elements in $\mathscr{P}_{\alpha}(A)$. Then there exist two basic open sets, say $D_{\alpha}(a)$ and $D_{\alpha}(b)$, such that $P \in D_{\alpha}(a), Q \in D_{\alpha}(b)$, and $D_{\alpha}(a) \cap D_{\alpha}(b)=\varnothing$. For any $K \in \mathscr{P}_{\alpha}(A)$, as $D_{\alpha}(a) \cap D_{\alpha}(b)=\varnothing$, the conditions $a \notin K$ and $b \notin K$ are not possible.

Case 1. If $a \in K$ and $b \notin K$, then $K \in D_{\alpha}(b)$.

Case 2. If $b \in K$ and $a \notin K$, then $K \in D_{\alpha}(a)$.

Case 3. If $a, b \in K$, then $K \in V_{\alpha}(\langle\{a, b\}\rangle)$.

In all cases, it holds that $K \in D_{\alpha}(a) \cup$ $D_{\alpha}(b) \cup V_{\alpha}(\langle\{a, b\}\rangle)$. This completes the proof.

Theorem 26. The following are equivalent:

(1) $\mathscr{P}_{\alpha}(A)$ is Hausdorff

(2) For any two distinct elements $P$ and $Q$ of $\mathscr{P}_{\alpha}(A)$, there exist $a, b \in A$ such that $a \notin P, b \notin Q$ and there does not exist any element $K$ of $\mathscr{P}_{\alpha}(A)$ such that $a \notin K$ and $b \notin K$

Proof. Suppose that $\mathscr{P}_{\alpha}(A)$ is Hausdorff and let $P$ and $Q$ be distinct elements of $\mathscr{P}_{\alpha}(A)$. Then there exist two basic open sets $D_{\alpha}(a)$ and $D_{\alpha}(a)$ such that $P \in D_{\alpha}(a), Q \in D_{\alpha}(b)$ and $D_{\alpha}(a) \cap D_{\alpha}(b)=\varnothing$. Now suppose on the contrary that there is $K \in \mathscr{P}_{\alpha}(A)$ such that $a \notin K$ and $b \notin K$. Then $K \in D_{\alpha}(a) \cap D_{\alpha}(b)$, which is absurd. Therefore, there does not exist any element $K$ of $\mathscr{P}_{\alpha}(A)$ such that $a \notin K$ and $b \notin K$. Conversely, assume (2). Let $P$ and $Q$ be two distinct elements in $\mathscr{P}_{\alpha}(A)$. Then, by assumption, there exist $a, b \in A$ such that $a \notin P$ and $b \notin Q$ and there does not exist any element $K$ in $\mathscr{P}_{\alpha}(A)$ such that $a \notin K$ and $b \notin K$, i.e., $P \in D_{\alpha}(a), b \in D_{\alpha}(b)$ and $D_{\alpha}(a) \cap D_{\alpha}(b)=\varnothing$. Thus, $\mathscr{P}_{\alpha}(A)$ is Hausdorff.

Theorem 27. $\mathscr{P}_{\alpha}(A)$ is regular if and only if, for any $P \in \mathscr{P}_{\alpha}(A)$ and $a \notin P(a \in A)$, there exist an ideal $I$ of $A$ and $b \in A$ such that $P \in D_{\alpha}(b) \subseteq V_{\alpha}(I) \subseteq D_{\alpha}(a)$.

Proof. Suppose that $\mathscr{P}_{\alpha}(A)$ is regular. Let $P \in \mathscr{P}_{\alpha}(A)$ and $a \notin P$. Then, $P \notin V_{\alpha}(a)$. As $\mathscr{P}_{\alpha}(A)$ is regular, there exist $\alpha$-ideals $I$ and $J$ of $A$ such that $P \in D_{\alpha}(I), V_{\alpha}(a) \subseteq D_{\alpha}(J)$, and $D_{\alpha}(I) \cap D_{\alpha}(J)=\varnothing$. Then, $\quad V_{\alpha}(J) \subseteq D_{\alpha}(a)$ and $D_{\alpha}(J) \subseteq V_{\alpha}(I)$. From $P \in D_{\alpha}(I)$, we have $I \nsubseteq P$. So we can choose $b \in I-P$. Now we show that $D_{\alpha}(J) \subseteq V_{\alpha}(b)$. If $K \in D_{\alpha}(J) \subseteq V_{\alpha}(I)$, then $I \subseteq K$ and hence $b \in K$. Thus, $K \in V_{\alpha}(b)$ and $D_{\alpha}(J) \subseteq V_{\alpha}(b)$. Taking their complement, we get $D_{\alpha}(b) \subseteq V_{\alpha}(J)$. Thus, for any $P \in \mathscr{P}_{\alpha}(A)$ and $a \notin P$, there exist an ideal $J$ of $A$ and $b \in A$ such that

$$
P \in D_{\alpha}(b) \subseteq V_{\alpha}(J) \subseteq D_{\alpha}(a)
$$

Conversely, suppose that the condition of the theorem is satisfied. To show that $\mathscr{P}_{\alpha}(A)$ is regular, let $P \in \mathscr{P}_{\alpha}(A)$ and 
$I$ be an $\alpha$-ideal of $A$ such that $P \notin V_{\alpha}(I)$. Then $I \nsubseteq P$. Choose $a \in I$ and $a \notin P$. By assumption, there exist an $\alpha$-ideal $J$ of $A$ and $b \in A$ such that

$$
P \in D_{\alpha}(b) \subseteq V_{\alpha}(J) \subseteq D_{\alpha}(a) .
$$

If we put $\mathscr{G}=D_{\alpha}(b)$ and $\mathscr{H}=D_{\alpha}(J)$, then $\mathscr{G}$ and $\mathscr{H}$ are disjoint open sets in $\mathscr{P}_{\alpha}(A)$ such that $P \in \mathscr{G}$ and $V_{\alpha}(I) \subseteq \mathscr{H}$. Thus, $\mathscr{P}_{\alpha}(A)$ is regular.

\section{Conclusion}

The study of the space of $\alpha$-prime ideals of universal algebras is initiated in the paper and it needs more investigation. It is observed by the author that it is promising to obtain a reticulation of a $c$-idempotent algebra $A$ induced by $\alpha$-ideals.

\section{Data Availability}

No data were used to support the results of this study.

\section{Conflicts of Interest}

The author declares that there are no conflicts of interest regarding the publication of this paper.

\section{References}

[1] W. H. Cornish, "Annulets and $\alpha$-ideals in a distributive lattice," Journal of the Australian Mathematical Society, vol. 15, no. 1, pp. 70-77, 1973.

[2] Y. S. Pawar and S. S. Khopade, "Ideals in 0-distributive lattices," Acta Universitatis Palackianae Olomucensis, Facultas Rerum Naturalium, Mathematica, vol. 49, pp. 63-74, 2010.

[3] G. C. Rao and M. S. Rao, " $\alpha$-ideals in almost distributive lattices," International Journal of Contemporary Mathematical Sciences, vol. 4, pp. 457-466, 2009.

[4] M. S. Rao, "Annulets and $\alpha$-ideals of C-algebras," AsianEuropean Journal of Mathematics, vol. 4, pp. 1-15, 2013.

[5] K. A. Mokbel, “ $\alpha$-\$ \alpha \$-ideals in \$0\$-distributive posets," Mathematica Bohemica, vol. 140, no. 3, pp. 319-328, 2015.

[6] C. Jayaram, "Prime $\alpha$-ideals in 0-distributive lattice," Indian Journal of Pure and Applied Mathematics, vol. 3, pp. 331-337, 1986.

[7] A. Bigard, "Sur les z-sous-groups, d'un groupe reticule," Comptes Rendus de l'Académie des Sciences-Series A, vol. 266, pp. 261-262, 1968.

[8] I. Chajda and R. Halas, "Annihilators in universal algebra," Contributions to General Algebra, vol. 14, pp. 29-34, 2004.

[9] G. M. Addis, "Annihilators in universal algebras: a new approach," Journal of Mathematics, vol. 2020, Article ID 1469727, 8 pages, 2020.

[10] S. Burris and H. P. Sankappanavar, A Course in Universal Algebra, Springer-Verlag, Berlin, Germany, 1981.

[11] G. Gratzer, General Lattice Theory, Academic Press, Cambridge, MA, USA, 1978.

[12] G. Gratzer, Universal Algebras, Van Nostrand, Princeton, NJ, USA, 1968.

[13] H. P. Gumm and A. Ursini, "Ideals in universal algebras," Algebra Universalis, vol. 19, no. 1, pp. 45-54, 1984.
[14] A. Ursini, "Prime ideals in universal algebras, acta universitatis carolinae," Mathematica et Physica, vol. 25, pp. 75-87, 1984.

[15] P. Agliano, "The prime spectrum of a universal algebra," Rivista di matematica pura ed applicata, vol. 4, pp. 83-91, 1989.

[16] P. Aglianò and A. Ursini, "Cosets in universal algebra," Journal of Algebra, vol. 107, no. 2, pp. 376-384, 1987.

[17] P. Agliano and A. Ursini, On Some Ideal Basis Theorems, Universita di Siena, Siena, Italy, 1988. 